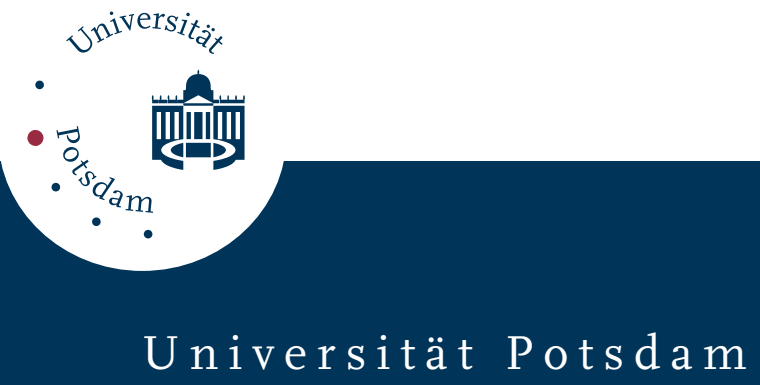

Margret Selting

\title{
Konstitution und Veränderung von Sprechstilen als \\ Kontextualisierungsverfahren : die Rolle von Sprachvariation und Prosodie
}

first published in:

Stil und Stilisierung : Arbeiten zur interpretativen Soziolinguistik / hrsg. von Volker Hinnenkamp und Margret Selting. - Tübingen : Niemeyer, 1989 ISBN 3-484-30235-6, S. 203-225

Postprint published at the Institutional Repository of the Potsdam University:

In: Postprints der Universität Potsdam

Philosophische Reihe ; 47

http://opus.kobv.de/ubp/volltexte/2010/4192/

http://nbn-resolving.de/urn:nbn:de:kobv:517-opus-41925

Postprints der Universität Potsdam

Philosophische Reihe ; 47 


\title{
KONSTITUTION UND VERÄNDERUNG VON SPRECHSTILEN ALS KONTEXTUALISIERUNGSVERFAHREN: DIE ROLLE VON SPRACHVARIATION UND PROSODIE ${ }^{1}$
}

\author{
Margret Selting
}

\section{EINLEITUNG}

Ich will mich in meinem Beitrag mit 'Sprechstilen' in Gesprächen befassen. Mit 'Sprechstil' will ich die sozial und interaktiv relevante (bedeutsame) Art des Sprechens in Situationskontexten erfassen (vgl. auch Sandig 1986:23, Hymes 1979:183). Ich will zeigen, wie im hier zugrunde gelegten Material, zwei Ausschnitten aus einem Gespräch zwischen einer Klientin und einem Beamten eines Sozialamts im Ruhrgebiet, insbesondere die Herstellung und Veränderung sprachlicher Varietäten im Gespräch und prosodische Signale neben sprachlichen Formulierungsverfahren auf der 'Textebene' verwendet werden, um Interaktionsmodalitäten und -rahmen zu signalisieren und Interaktion fortlaufend gegebenenfalls neu zu kontextualisieren.

\section{GRUNDLEGENDE ANNAHMEN}

Ich gehe davon aus, daß Interaktionspartner zu Beginn einer Interaktionsgeschichte und gegebenenfalls zu Beginn oder an entscheidenden Stellen innerhalb eines Gesprächs erneut einen situativ bzw. für bestimmte Aktivitäten unmarkierten Referenzsprechstil als für die anstehende Interaktion 'normalen', 'unmarkierten', 'unauffälligen' Sprechstil aushandeln bzw. etablieren. Diese Wahl bzw. Aushandlung läßt aber gerade auch Spielraum für lokale Variation oder auch spätere Umdefinitionen oder Neu-Aushandlungen des Referenzstils. Innerhalb derselben Situation orientieren sich dann die Gesprächspartner retrospektiv am Referenz- und am vorher verwendeten Sprechstil als tertium comparationis und prospektiv an den antizipierten Folgen und Wirkungen bei der lokalen Konstitution und Veränderung des Sprechstils.

Bei der durchzuführenden konversationellen Aktivität wird die Herstellung und Veränderung von Sprechstilen im Gespräch als Kontextualisierungsverfahren verwendet (vgl. Gumperz 1982, Auer 1986; vgl. auch Sandigs Konzept von Stil als 'Indexikalisierung' abstrakter Schemata, 1986:31f und 34), im vorliegenden Fall zur Signalisierung und Herstellung lokaler Interaktionskontexte, die ich als Interaktionsrahmen und -modalitäten analysiere und die jeweils unterschiedliche Interpretationsschemata nahelegen. Gerade

1 Für hilfreiche Kommentare zu einer früheren Version dieses Papers danke ich v.a. Peter Auer und Volker Hinnenkamp. 
deren Ausnutzbarkeit zeigt jedoch hier die Übergeordnetheit des globalen behördlichen Rahmens, an dem sich die Interaktionspartner orientieren, und der hierin erwarteten sozialen und interaktiven Verhältnisse, die sie selbst durch ihr Handeln reproduzieren.

\section{DAS BEISPIELMATERIAL}

Die folgenden Gesprächsausschnitte stammen aus einem Gespräch zwischen einem Mitarbeiter des Sozialamts B und einer Klientin K in einem Dienstzimmer eines Sozialamts im westlichen Ruhrgebiet (aus dem Sonderkorpus "Sozialamtsgespräche" des IdS Mannheim). Anlaß dieses Gesprächs war, da $B$ - wie aus dem Gesprächsverlauf hervorgeht - B die K aufs Amt bestellt hat, um sie über ihren Aufenthaltsort zu befragen. Denn $\mathrm{K}$ hat auf Kosten des Sozialamts eine neue Wohnung gemietet, in der sie sich aber nicht aufhält. Das folgende erste Transkript setzt ein, als B nach einer längeren regelrechten Verhörphase, in der er $\mathbf{K}$ auch zum Weinen brachte, nun offenbar eine kooperativere Gesprächsphase einleiten will, in der die Besprechung der Problemlösung im Zentrum stehen soll, nämlich die von $\mathrm{K}$ zu unternehmenden Schritte für ihren Einzug in ihre neue Wohnung. In den Folgeturns schlägt B einen scheinbar zunehmend 'informelleren Ton' an und elizitiert schließlich eine Art Tratscherzählung von K. Unmittelbar im Anschluß an K's Erzählung jedoch dreht er "den Spieß wieder um" und greift erneut K's Lebenswandel an. Retrospektiv entsteht der Eindruck, zumindest B's Initiation der Tratscherzählung sei als strategischer Zug zu interpretieren.

\section{Transkript 1: WAT WAR DENN MIT DER HOCHZEIT}

(S-31-6: Band Nr. 2/1, Laufnr. 346-360)

347 B: |(relativ laut) also IRgndwie müssp wa doch mal

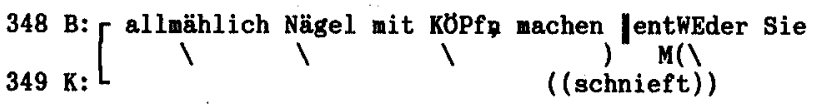

350 B: bezIEHN hier Ihre wOHnung (1.2) |ä:h*

$351 \mathrm{~K}$ : Tich НАВ Ih doch letzmal schon gesacht wenn se

352 so weit is ich zieh LIE:byd gern da ein* $1)-$

353 B: [T ITA: |Tda MÜSsq $\mathrm{S}$ sich aber ganz klein wenich $354 \mathrm{~K}:$ H(\) H( ((schnieft)) 
355 B: drum KüMmern*

$356 \mathrm{~K}:$ ((schnieft)) (0.8) |na ich Thab mi auch gehn lassn* H(I

$357 \mathrm{~K}$ : Jun da |DAmals wars mit Herrn GG F(/ V)

$358 \mathrm{k}$ - (sehr schnell) er hat Ihq ja jeSACHT wenn die*

$359 \mathrm{~K}:\left[\begin{array}{cc}(0.5) & \text { |aber dann } \\ & \text { jaa: } \\ (\backslash)\end{array}\right.$

361 K: |dann hattp se am DRITt Jubiläum

$$
F(T) \quad V)-
$$

$362 \mathrm{~K}:$ \|die hattp am SIEBmstq Jubiläum

$$
F(T) \quad \backslash)-
$$

$363 \mathrm{~K}:$ \|die hattq am $\underset{F(T)}{\text { ZEHnt Jubiläum }}$

$364 \mathrm{~K}:\left[\begin{array}{cc}\text { |und INge WaRte mal } \\ \mathrm{F}[(\backslash)\end{array}\right)\left(\begin{array}{l}\text { Iwat war denn } \\ \end{array}\right.$

366 B: mit der HOCHzeit \|wo die Frau GG mim: BROTmesser

$367 \mathrm{~B}:[$ angeblich: irgndwo los gegangn sein soll

$368 \mathrm{~K}$ :

(ANgeblich

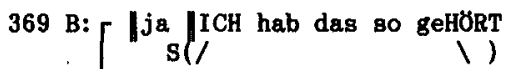

$370 \mathrm{~K}:\left[\begin{array}{c}\text { S( } \\ \text { I(chnell) also ich* ! }\end{array}\right.$

$371 \mathrm{~K}$ : (laut) also ICH SA:ch da NIX zu* aber . ä $M(1) \backslash 1-$

$372 \mathrm{~K}$ : WIR wissen von unsern HAUSwirt (von Herrn EE .

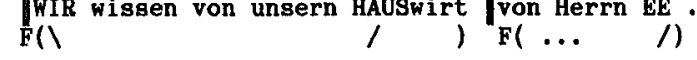


$373 \mathrm{~K}:\left[\begin{array}{c}\text { (sehr schnell, leiser) naja un DER muß es ja WISsn* } \\ 374 \mathrm{~S}(/\end{array}\right.$

$375 \mathrm{~K}$ : Uda war POLteramd von seiner TOCHter. F( $1 /$ )

$376 \mathrm{~K}$ : Un da hat ihr MANN wohl mit ner ANdern geTANZT F(I

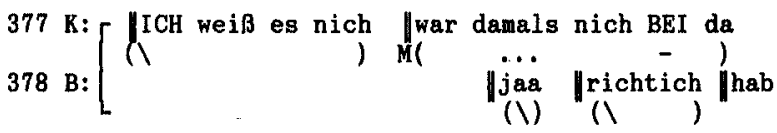

$379 \mathrm{~K}:[$ - $\|($ rel. laut) GING se L0:S*

380 B: $\left[\begin{array}{c}\text { ich geHorT } \\ (\backslash)\end{array}\right.$

$381 \mathrm{~K}$ : Un dann HAT $\mathrm{s}$ sich mit: ihrm MANN wohl AUCH gehabt M( 1 , )-

$382 \mathrm{~K}:$ |un dann m $\ddot{a}: h$

(schneller $u$. bis * immer leiser werdend)

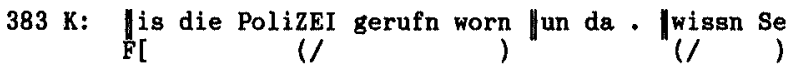

$384 \mathrm{~K}: \|^{\mathrm{i}}$ is son KLEInes Haus $\|$ da geht die TREPpe so steil hoch

$385 \mathrm{~K}:$ Un da hat se wohl gestandn nur Jun kein Schritt WEI ter $(1)$ ( )

$386 \mathrm{~K}$ : Uun dann ham se se nahher MITgenomm

$(1)$

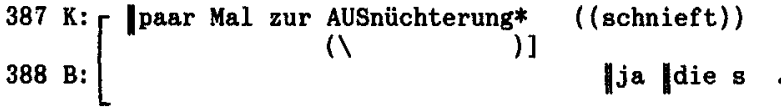

389 B: Idie SOLL auch sehr eifersüchtich auf SIE sein ne s( 1 i $) /$

390 B: $[(0.9)$ die Frau GG ne

$392 \mathrm{~K}$ : |ich hab EHRlich nichs (lachend) aso*

$$
\text { (1) }
$$


.393 K: ((schnieft zweimal, ca 1.3 Sekunden))

394 K: I(laut) seh ich WIRKlich danach aus daß ich daß ich

$$
\text { F() }
$$

$395 \mathrm{~K}:$ n MANnerwamp bin

1 )-

Das folgende zweite Transkript gibt eine spätere kooperativere Phase in diesem Gespräch wieder, in der $\mathbf{B}$ der $\mathbf{K}$ genauere Instruktionen zur Beschaffung von Einrichtungsgegenständen gibt, damit $\mathrm{K}$ dann endlich in ihre Wohnung einziehen kann.

Transkript 2: DER HERD

(S-31-6: Band Nr. 2/1, Laufnr. 390-400)

573 B: DER hat für SIE son: - son HERD

$574 \mathrm{~K}:$ [ (schnell) |ja wissn se |die ham HEI*

575 B:

$1(/$

GASherd

$576 \mathrm{~K}$ : HDIE ham HEI zung geKRICHT

F[F(

$577 \mathrm{~K}$ : [ un ham ALles RAUSgeworfn . dann lne

578 B: $\left[\begin{array}{c}F(\backslash \text { und und (?den HERD den?) könn SIE jetz brauchn } \\ \text { F( }(/)\end{array}\right.$

(1.0)

579 B: Jjaa DER verschenkt die nich (0.9)

580 B: Iäh der AA is ja froh wenn die hier sind hadder mir F(\ \ )-

581 B: gesacht .

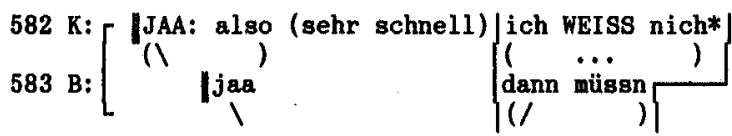

$584 \mathrm{~K}:$ (laut) ich HAB |ich HAB:

(I). (I) 
$585 \mathrm{~K}:$ (laut) also ich muß EHRlich sagn ich ich hab nich $F()$

$586 \mathrm{~K}$ : ich könnt ja SAGN er will jeld* aber ich:

$587 \mathrm{~K}$ : ich hab da NICH nach gefracht (1.1) $F(\backslash \backslash)$

588 B: Jjojo Ida BRAUchn se au nich

589 B: Idat ding könn se so mitnehm.

590 B: $H$ HADder gesacht (1.0)

591 B: |ä: hm |(schnell) THAM wa auf BAND $\mathrm{H}, \mathrm{F}(\backslash \backslash 1)$

$(2.0)$

592 B: [äh . JA |kann ich das SO machn

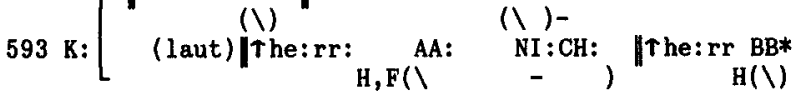

(1.1)

594 B: |jaJA nun

595 B: | (schnell) der AA hat dat aber ANgegebm* $F(/$

$596 \mathrm{~K}:$ (leise) hwhm .

$597 \mathrm{~K}:$ [ (leise) muser wehr wissen als ich*

598 B:

F( \\)

(leise) wir WISsen dat jednfalls*

() )

$(0.8)$

599 B: is auch kein proble:M

(1)

600 B: |wenn sie jetz also MEHrere GEggstände habm

601 B: Idie jetz (0.7) 
$602 \mathrm{~K}: \quad \stackrel{\mathrm{mhm}=}{\mathrm{V}}$

603 B: $[$ =äh die sie BRAUchen und die sie kriegn könn

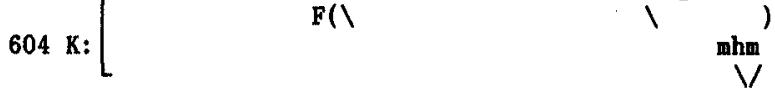

605 B: [KOStalos oder gegn geringn PREIS

$606 \mathrm{~K}:\left[\begin{array}{ll}\mathrm{M}(\mathrm{mhm} & \mathrm{mhm}\end{array}\right.$

607 B: [ (leiser) dat müssn wa dann no AUShandeln* .

$608 \mathrm{~K}$ :

609 B: Udann RUF ich den EILbotn an

s() $/$ )

610 B: Un und SAGE wir sind beREIT die und die $\mathrm{s}(1$

611 B: [ KOStq für den transPORT zu überneh廿 (1.1)

$612 \mathrm{~K}:$

((atnet laut aus))

613 B: Vaber ich KANN nich für EI\$ HERD den $\mathrm{M}(1) /$

614 B: EILbot DAhin schicken

$(1)$ )

615 B: Un dann kow se nächste mal mit n STUHL H(l $/$ )

$616 \mathrm{~B}$ : Uun den hol ich dann in HaLstade mit $\mathrm{n}$ eilbotq (0.7) F()

$617 \mathrm{~K}$ : JETZ hab ich das kapiert

(1)

\section{ANALYSE}

Zunächst stelle ich die Kategorien zur Beschreibung der hier konstituierten Sprechstile und deren Variation vor; danach versuche ich eine Rekonstruktion der mit der Konstitution und Veränderung von Sprechstilen produzierten interaktiven und sozialen Bedeutungen. 


\subsection{BESCHREIBUNGSKATEGORIEN}

Im folgenden werden als sprechstilkonstituierende Mittel neben den für bestimmte Aktivitäten wie z.B. Erzählungen und Instruktionen verwendeten sprachlichen Formulierungsweisen vor allem die Signalisierung und Veränderung sprachlicher Varietäten und prosodische Signalisierungsmittel berücksichtigt. Bezüglich dieser Mittel sind einige Vorbemerkungen nötig.

Zunächst zu den sprachlichen Varietäten: Im hier zugrunde liegenden Gespräch haben $B$ und $\mathrm{K}$ seit Gesprächsanfang ein Spektrum an Sprachvariation als situativ unmarkierte Referenzvarietät etabliert, das ich als 'unmarkierte Umgangssprache'2 bezeichne (vgl. hierzu auch die Beschreibung der Anfangsphase dieses Gesprächs in Selting 1989a). $\mathrm{Zu}$ dieser unmarkiert umgangssprachlichen Varietät kommen die Gesprächspartner im Laufe des Gesprächs bei kooperativen Sequenzen immer wieder zurück, während sie in antagonistischen und strategischen Sequenzen ihre signalisierten Varietäten in Richtung auf Pole hin verändern, die ich als 'hochsprachlich'3 und 'markiert umgangssprachlich' bzw. 'ruhrdeutsch' (vgl. Mihm 1985) bezeichne. Allerdings sind beide Sprecher offenbar keine native speaker des Ruhrdeutschen ( $\mathrm{K}$ ist ursprünglich Berlinerin!); ein "echtes" Ruhrdeutsch erreichen sie nie und scheinen es auch gar nicht anzustreben. Aus diesem Grunde verwende ich für die von den Partnern konstituierten Annäherungen an das Ruhrdeutsche mithilfe auffälliger (stereo-) typischer Formen den unschönen Begriff 'markierte Umgangssprache’5. Varietätenveränderungen im Gespräch werden angezeigt durch

2 Für eine genauere Analyse der Sprachvariation in diesem Gespräch und bezüglich der Zuordnung einzelner Merkmale siehe Selting 1989.

3 Siehe Fußnote 4.

4 Vgl. zu auffälligen, primären, und unauffälligen, sekundären, dialektalen Merkmalen und deren Wandel/Abbau im Sprachkontakt insbesondere auch Schirmunski 1930 und Reiffenstein 1976, 1980. Gegenüber Reiffensteins Erklärung des Unterschieds zwischen primären und sekundären Unterschieden zwischen Hochsprache und Mundart auf der Basis der GTG (primäre Unterschiede als Resultat unterschiedlicher TS-Lexikoneinträge, sekundäre Unterschiede als Resultat phonologischer Regeln bei derselben TS), ist m.E. Schirmunski's ältere "sprachpsychologische" Erklärung für den hier vertretenen interpretativen Ansatz erklärungsstärker: Da die primären Merkmale auffälliger und damit 'bemerkbarer' sind, können sie leichter bewußt oder intuitiv abgebaut oder aber auch methodisch-systematisch als stereotype Signale verwendet werden (vgl. Schirmunski 1930:183ff.). (Den Hinweis auf die Arbeiten von Schirmunski und Reiffenstein verdanke ich Peter Auer.)

5 Die von den Gesprächspartnern konstituierten Sprachvarietäten sollten weder als eindeutig gegeneinander abgrenzbare Einheiten noch als nicht weiter in sich strukturiertes Kontinuum zwischen zwei Polen aufgefaßt werden. Zumindest im vorliegenden Fall, in dem die Sprecher ein eher norddeutsches Varietätenspektrum konstituieren, bewegen sie sich innerhalb eines in sich oder intern strukturierten Kontinuums von einer quasi situativ unmarkierten Referenzvarietät in Richtung auf zwei entgegengesetzte und gegenüber der Referenzvarietät markierte Pole zu und zurück. Die Referenzvarietät und die beiden Pole werden offenbar als typisierte und dennoch dynamische Konstrukte zugrunde gelegt bzw. 'angesteuert'; die interne Struktur der Pole läßt sich wiederum modellieren als Kern von besonders typischen Merkmalen und Rand/Peripherie mit weniger typischen, unzuordbaren oder in geringer Dichte auftretenden nicht-typischen Merkmalen anderer Varietäten. Dies legt ein Modell nahe, in dem zwischen einem markierten Kern und einer unmarkierten Peripherie von 
entweder auffällige Veränderungen der Dichte von Formen für eine bestimmte Varietät und/oder durch die Verwendung besonders auffälliger und typischer Kernmerkmale für diese Varietät. Die im Gespräch verwendeten sprachlichen Varietäten bzw. die verwendeten sprachlichen Merkmale und deren Variation interessieren jedoch hier nicht als oder im Hinblick auf linguistische Subsysteme innerhalb des Varietätensystems des Deutschen. Hier werden sie vielmehr als sozial und interaktiv bedeutsame Konstruktionen, mithin als (Konstituenten von) 'Sprechstile(n)' oder als Stilmittel aufgefaßt, die auf bestimmte Interpretationsrahmen verweisen und damit bestimmte Interpretationen von Äußerungen nahelegen. (Vgl. hierzu auch Dittmar/Schlobinskis 1988 Analyse der Sprachvariation in Gesprächen aus dem Berliner Raum.)

Nun zur Prosodie: Im Bereich der Prosodie werden hier vor allem Sprechgeschwindigkeit und Lautstärke, Intonation bzw. genauer globale Tonhöhenverläufe auf ganzen Äußerungseinheiten und lokale Tonhöhenverläufe auf akzentuierten Silben (Akzenttypen), und unterschiedliche Akzentrhythmen als Signalisierungsmittel verwendet.

Bei der Sprechgeschwindigkeit kontrastiert schnelles mit normalem Tempo, bei der Lautstärke normale mit lauterer.

Intonation bzw. globale und lokale Tonhöhenveränderungen und unterschiedliche Akzenttypen (vgl. Selting 1987 und verbessert 1989a) werden verwendet zur Signalisierung von kohäsiven Anknüpfungsbeziehungen und zur Signalisierung der internen Struktur von Redebeiträgen.

Beim Akzent-Rhythmus (vgl. auch Bolinger 1986:63ff) werden offenbar kürzere bzw. 'normal' lange Akzenteinheiten mit merklich längeren Akzenteinheiten kontrastiert. Als Akzenteinheit bezeichne ich dabei die Einheit beginnend mit einer Akzentsilbe, die in der Intonationszeile der Transkripte innerhalb von Klammern mit einem 'Y', '/' oder '-' für verschiedene Akzenttypen notiert ist, mit den folgenden nicht bzw. schwach akzentuierten Silben und gegebenenfalls rhythmischen Pausen bis zur nächsten Akzentsilbe, diese aber nicht einschließend. ${ }^{6}$ Die Länge bzw. Dauer einer Akzenteinheit läßt sich einerseits, nach

Merkmalen als der internen Struktur typisierter Varietäten in Gesprächen unterschieden wird. Hierzu paßt dann v.a. auch die Unterscheidung zwischen auffälligen primären und unauffälligen bzw. weniger auffälligen sekundären Merkmalem wie in Anmerkung 4 dargelegt. Vgl. genauer hierzu auch Selting 1989.

6 Die hier angenommene Akzenteinheit unterscheidet sich von der Einheit des Taktes oder Fußes, die üblicherweise bei der Beschreibung von Rhythmus zugrunde gelegt wird. Takte bzw. Füße sind - soweit ich z.B. Abercrombie 1964:217, Halliday 1967:12f oder Pheby 1980: 46ff verstehe - die Einheiten, die aus einer auf der Wortebene betonten/starken (im Englischen 'stressed' bzw. bei Halliday 'salient') Silbe und den folgenden unbetonten/schwachen ('unstressed') Silben bestehen. Der hier vorfindbare Rhythmus scheint z.T. Bolingers 'syllabic rhythm' zu ähneln, bei dem Einheiten aus einer vollen Silbe plus den folgenden reduzierten Silben relevant sind (1986:39, 63). Diese 'stressed/salient syllables' müssen jedoch nicht auf der Satz- oder Äußerungsebene akzentuiert sein; theoretisch sind vielmehr 'stressed syllables' auf der Wortebene (potentiell) akzentuierbare Silben auf der Satz- bzw. Äußerungsebene, nicht jedoch unbedingt auch tatsächlich akzentuierte. In Bezug auf diese Einheiten zwischen betonten/starken Silben, also aus 'stressed syllable' mit folgenden 'unstressed syllables', wurde auch $\mathrm{m}$.W. das Problem der Isochronie bisher vornehmlich diskutiert. Diese Einheiten scheinen jedoch i.d.R. kleiner zu sein als die hier zugrunde gelegte Akzenteinheit. (Laut Pheby besteht ein Takt im Deutschen aus einer starken Silbe und "meist bis $4^{\text {n }}$ schwachen Silben.) 
Festlegung der genauen Meßstelle, als rein physikalische Zeitmessung mit einem geeigneten Meßgerät erfassen; andererseits lassen sich längere Akzenteinheiten aber auch intuitiv im Vergleich zu vorhergegangenen kürzeren Einheiten z.B. als ungefähr doppelt so lang o.ä. grob bestimmen. Die Abfolge von Akzenteinheiten konstituiert den Akzentrhythmus. Ein Rhythmus ist 'regelmäßig-isochron' bzw. kurz 'regelmäßig', wenn aufeinanderfolgende Akzenteinheiten ungefähr gleich lang sind, sonst ist er 'unregelmäßig'. Der Akzentrhythmus kann gegebenenfalls über Konturengrenzen hinweg z.B. Nach- und Vorläufe aufeinanderfolgender Konturen oder auch aufeinanderfolgende Beiträge unterschiedlicher Sprecher über einen Sprecherwechsel hinweg als eine Einheit signalisieren; er kann aber auch durch Verzögerungen o.ä. unterbrochen werden, wenn Sprecher quasi 'aus dem Rhythmus' geraten. In den vorliegenden Gesprächsausschnitten werden offenbar kürzere Akzenteinheiten mit einer Dauer von i.d.R. weniger als ca. 0.8-1.0 Sek. kontrastiert mit längeren, meist ca. doppelt oder ơreifach so langen Akzenteinheiten mit mehr als ca. 1.0 Sek. Dauer. Diese werden ebenso wie die Variation segmentaler Mittel als bedeutsame Stilmittel verwendet.

In den unten stehenden Transkriptausschnitten orientiert sich die Zeilenschreibweise an Akzenteinheiten. Die am Rand angegebene Dauer der Einheit in Zehntelsekunden wurde mit einer Stoppuhr manuell ermittelt, da die Signalqualität des Materials für eine instrumentelle Analyse nicht ausreichte. Bei unterschiedlichen Meßwerten bei mehreren aufeinanderfolgenden Messungen wurde ein Mittelwert notiert. Diese Dauerangabe kann deshalb auch nur als äußerst grober Vergleichswert interpretiert werden, der hier nur die auditive Analyse etwas erhärten und stützen soll.

Ein im vorliegenden Fall suggestives Bild ergibt auch eine Zählung der Silben in den Akzenteinheiten; diese liegt nämlich bei kurzen Akzenteinheiten bei bis zu ca. 6, bei längeren hingegen bei bis zu 14. Da jedoch das Deutsche hinsichtlich seiner rhythmischen Struktur üblicherweise nicht zu den silben-, sondern zu den akzentzählenden Sprachen gezählt wird, in denen es demzufolge keine eindeutige Beziehung zwischen der Anzahl der Silben und der rhythmischen Struktur der Rede gibt, ermöglicht eine solche Zählung allenfalls eine erste suggestive Orientierung, kann aber nur schwerlich als Basis einer genaueren Analyse gelten.

Im folgenden will ich nun darstellen, wie die beiden sprechstilkonstituierenden

Im Unterschied hierzu gibt es bei Bolinger jedoch den 'accentual rhythm', bei dem die Einheit aus Akzentsilbe plus nachfolgenden unakzentuierten Silben grundlegend ist (ebd.:63ff). Weiterhin erscheinen in neueren Arbeiten wie bei Erickson/Shultz 1982 als für den Rhythmus relevante Einheit die Tongruppe (ebd.:75, 85ff): der zeitliche Abstand der 'stressed tonal nuclei', "most distinctly marked by a combination of pitch (intonation) shifts and increased loudness (stress)" (ebd.:75), sei oft gleich lang. Auch Gumperz 1982 (109ff) impliziert offenbar eine Variation der Tongruppenlänge als interaktiv relevant. Der Unterschied zwischen 'stressed tonal nuclei' bei Erickson/Shultz und 'accents' bei Bolingers 'accentual rhythm' als Aufhängepunkten für die Beschreibung des Rhythmus scheint mir allerdings nicht sehr groß zu sein, da Erickson/Shultz anscheinend mehr als die Nuclei im Sinne einer strengen tonetischen Analyse berücksichtigen und sich damit offenbar an eine Analyse wie die Bolingersche Akzentanalyse annähern. Die von Erickson/Shultz und insbesondere von Bolinger als 'accent feet' betrachteten Einheiten entsprechen wohl am ehesten dem, was ich hier 'Akzenteinheit' nenne. Diese 'Akzenteinheit' scheint sich ebenfalls weitgehend mit der von Couper-Kuhlen/Auer 1988 als 'Kadenz' ('cadence') bezeichneten Einheit zu decken. 
Signalisierungsmittel Sprachvariation und Rhythmus im vorliegenden Gesprächsausschnitt kookkurrierend miteinander und mit bestimmten Formulierungsverfahren als Kontextualisierungshinweise verwendet werden.

\subsection{ANALYSE DER INTERAKTIVEN HERSTELLUNG UND FUNKTION VON SPRECHSTILVERÄNDERUNGEN IM GESPRÄCHSVERLAUF}

In den vorliegenden Gesprächsausschnitten verändern die Gesprächspartner in relativ schneller Abfolge ihre Sprechstile und signalisieren und konstituieren damit spezifische lokale Interaktionskontexte, nämlich unterschiedliche Interaktionsmodalitäten und -rahmen $^{7}$, in denen dann auch jeweils unterschiedliche Interpretationsschemata naheliegen.

Zunächst zum ersten Gesprächsausschnitt:

In Z. 347ff. beginnt B mit einer relativ hochsprachlichen Varietät und verschiebt diese im Laufe der Sequenz zunächst immer weiter in Richtung unmarkierter und schließlich markierter Umgangssprache. In Zeile 347-350 leitet er eine kooperativere Gesprächsphase ein, in der er auf zukünftige Aktivitäten der $\mathrm{K}$ und eine generelle Lösung des Konflikts zwischen dem Sozialamt und $\mathrm{K}$ fokussiert. Mit der relativ hochsprachlichen Varietät signalisiert er noch eine formelle Interaktionsmodalität, in der die Äußerung nur im Rahmen institutioneller Interpretationsschemata als begonnene und von $K$ unterbrochene Drohung interpretiert werden kann. Dennoch scheinen insbesondere die zahlreichen Heckenausdrücke und Partikeln, die Redewendung Nägel mit Köpfn machen und der noch unregelmäßige Rhythmus im Vorlauf der Drohung in Z. 347-348 trotz der vielen hochsprachlichen Formen in der Äußerung die Drohung selbst abzumildern und ein eher kooperatives Entgegenkommen an $\mathrm{K}$ zu signalisieren; zugleich wird die Problemlösung nun auch als gemeinsame Aktivität präsentiert (siehe wa in Zeile 347). Die Drohung selbst erscheint dann mit auditiv prägnanten kurzen Akzenteinheiten: ${ }^{8}$

WEder sie be

ZIEHN hier Ihre

WOHnung
(0.6)

7 Vgl. hierzu auch Müller 1984:106ff. Mit 'Interaktionsmodalitäten' will ich hier den Unterschied zwischen einer eher formellen und einer eher informellen Interaktionsweise erfassen, mit 'Interaktionsrahmen' den Unterschied zwischen z.B. 'institutionell-behördlich' und 'alltagsweltlich' (vgl. hierzu Selting 1985).

8 Die Darstellungsweise lehnt sich an diejenige von Erickson/Shultz 1982 an, meine Zeitmessung ist jedoch viel ungenauer. Im Unterschied zu Erickson/Shultz geht es mir hier auch nicht um die Analyse der gemeinsamen Etablierung eines Interaktionsrhythmus zwischen den Interaktionspartnern, sondern nur um die Herstellung und Veränderung von Sprechrhythmen bzw. Akzentverteilungen in aufeinanderfolgenden Äußerungen als Kontextualisierungshinweisen. 
$\mathrm{K}$ bezieht sich dann auf ihre und B's gemeinsame Interaktionsgeschichte und beteuert, ihre neue Wohnung beziehen zu wollen, wenn se so weit is. Hierbei führt sie B's bisherige Varietätenverschiebung weiter fort in Richtung unmarkierter Umgangssprache und verwendet hohe globale Tonhöhe und einen unregelmäßigen Rhythmus mit einer sehr langen Akzenteinheit:

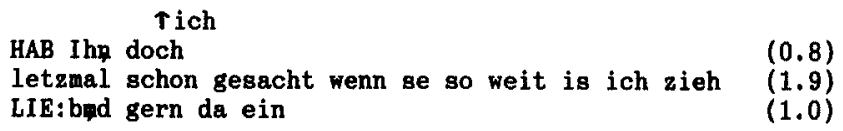

Genau hieran knüpft B dann in Z. 353-355 an und beschuldigt K der Inaktivität. Obwohl aber $\mathbf{B}$ der $\mathrm{K}$ in dieser Äußerung mit regelmäßig kurzen Akzenteinheiten einen Vorwurf macht, signalisiert er dennoch eine informelle Interaktionsmodalität, und zwar durch die Wahl der Verniedlichungsform ganz klein wenich, durch die weitere Varietätenverschiebung in Richtung auf eine markierte umgangssprachliche Sprachvarietät und durch seine global hohe Tonlage, die eine Anknüpfung an K's vorherige global hohe Tonlage herstellt. Insgesamt wird der Ton lebhafter und weniger autoritär.

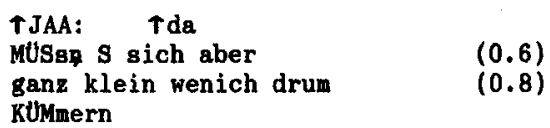

Entsprechend reagiert $\mathrm{K}$ auch nicht wie im vorherigen "Verhör" mit einer bloßen Selbstverteidigung, sondern gesteht $\mathrm{zu}$, sich gehen gelassen $\mathrm{zu}$ haben und schildert die Hintergründe ihrer vermeintlichen Inaktivität. Dabei bleibt sie bei der unmarkierten Umgangssprache und bei einer eher lebhaften Intonation, zunächst aber mit ziemlich unregelmäßigem Rhythmus. Ab Z. 361 signalisiert sie einen Übergang zu erzählender Rede, und zwar vor allem durch einen Wechsel zu sehr regelmäßigen Rhythmen mit kurzen Akzenteinheiten und ebenfalls rhythmischen lokalen Tonhöhensprüngen und durch einen gleichzeitigen Wechsel zur Verwendung formelhafter Parallelkonstruktionen in einer Erzählung, in der sie sich selbst als "Objekt" darstellt:

$\begin{array}{ll}\text { dann hattn se am } & (0.6) \\ \text { DRITt Jubi } & (0.6) \\ \text { läum die hatt am } & (0.8) \\ \text { SIEbmstp Jubi } & (0.6) \\ \text { läum die hattp am } & (0.8) \\ \text { ZEHNt Jubi } & (0.6) \\ \text { läu und } & (0.5) \\ & \\ \text { INge } & (0.3) \\ \text { WARte mal } & (0.5) \\ \text { Inge } & (0.3) \\ \text { WARte mal } & (0.4)\end{array}$

Diese Erzählung führt damit die von B initiierte informelle Interaktionsmodalität fort (vgl. insbesondere Quasthoff 1980: Kapitel 2.3. zur rahmensetzenden Funktion von Erzählungen in Sozialamtsgesprächen). 
Anknüpfend an K's Erwähnung von Feierlichkeiten fokussiert B dann in Zeile 365367 eine spezifische Hochzeitsfeier und ein spezifisches Ereignis: Indem er dieses Ereignis, in das eine dritte, beiden bekannte Person verwickelt war, in seinem Höhepunkt benennt und zugleich sein Wissen darüber als "aus zweiter Hand" und vage darstellt, initiiert er hier offenbar eine 'Klatsch-' oder 'Tratscherzählung'. Dabei verwendet er die ruhrdeutsche Form wat (Zeile 365) neben lauter anderen umgangssprachlichen Formen auf morphophonemischer, syntaktischer (wo) und lexikalisch-idiomatischer Ebene (mim Brotmesser auf jemanden losgehen); im ganzen Transkript ist dies die Stelle, an der B sich dem ruhrdeutschen Pol am weitesten nähert. Gleichzeitig wird der Ton 'beiläufiger': Anstelle ziemlich kurzer Akzenteinheiten in seinen vorherigen Beiträgen läßt B in Zeile 366f seine Äußerung über die lange Strecke von 2.37 Sekunden hinweg nur schwach akzentuiert auslaufen:

$\begin{array}{lr}\text { wat war denn mit der } & (0.6) \\ \text { HOCHzeit wo die Frau } & (0.9) \\ \text { GG mim: } & (0.9) \\ \text { BROTmesser angeblich: irgndwo los gegangn sein soll } & (2.4)\end{array}$

Weiterhin tritt an die Stelle direkter Anrede der K vorher (vgl. Z. 348 und 353) und nachher (siehe Z. 389) hier eine indirekte Adressierung, indem B ihr die Erfüllung einer konditionellen Relevanz auferlegt; offenbar wird sie hier nicht mehr als Klientin angesprochen. Sowohl thematisch wie auch sprechstilistisch wird hier also deutlich eine Weiterführung der informellen Interaktionsmodalität und konkreter noch ein Rahmenwechsel zur alltagsweltlichen "Unterhaltung" hin signalisiert.

Dieser Rahmenwechsel wird von K dann ratifiziert und sie liefert in Zeile 370-387 eine Erzählung mit formelhaften und z.T. tratschähnlichen Zügen'.

Nachdem sie sich nach der spontanen Erstreaktion $A$ ggeblich zunächst laut von der Rolle der 'Tratschenden' distanziert, stellt sie sich dann mit veränderter Prosodie als Mitglied einer 'Wir-Gemeinschaft' dar, die die thematisierte Geschichte aus zuverlässiger Quelle wei $\beta^{10}$. In der Gesamterzählung selbst werden dann offenbar, die unsichereren Elemente mit der Partikel wohl angezeigt und so von den sichereren Elementen un-

9 Kallmeyer/Keim 1986 heben u.a. die Formelhaftigkeit von Formulierungen im Tratsch hervor, die in der von ihnen untersuchten Gruppe Mannheimer Frauen zur Kontextualisierung und Symbolisierung sozialer Identität verwendet wird. Weiterhin weisen sie auf die Vielgestaitigkeit von Tratschereignissen hin, deren Gegenstand das 'Reden über die Welt' ist, das dadurch zustandekommt, daß sich "das Interesse primär auf unterschiedliche Aspekte des Wissens über die Welt und des Redens über dieses Wissen richten kann" (ebd.:104). In der vorliegenden Erzählung der $\mathbf{K}$ handelt es sich wohl um den Aspekt der 'Faktizität', "also darum, wie etwas ist/war bzw. ob es wirklich so ist/war" (ebd.), der von B mit der Formulierungsweise angeblich ... sein soll ja auch fokussiert worden war. Streeck (1985:58f., Anmerkung 40) gibt als allgemeine Definition für Klatsch und Tratsch folgende: "Klatsch und Tratsch läßt sich im weitesten Sinne als eine verbale Aktivität charakterisieren, in denen zwei Personen über eine ihnen gemeinsam bekannte, abwesende, dritte Person Informationen austauschen, an deren Verbergung diese interessiert ist, da sie ihr öffentlich präsentiertes 'Image' diskreditieren könnten". Am ausführlichsten hierzu: Bergmann 1987.

$10 \mathrm{~K}$ 's Intonation signalisiert dabei wiederum Anknüpfungsbeziehungen. Auf die genaue Rolle der Intonation kann ich hier nicht näher eingehen. 
terschieden. In Z. 377 weist K noch einmal explizit ihr Wissen als "aus zweiter Hand" aus, woraufhin B ebenfalls explizit die Richtigkeit ihrer Darstellung bestätigt.

In der Gesamterzählung folgt $\mathrm{K}$ auch B's vorheriger Varietätenverschiebung in Richtung Ruhrdeutsch: Es finden sich nur wenige hochsprachliche Formen (wir wissen, Zeile 372, auch, Zeile 381, also, Zeile 371), sehr zahlreiche unmarkiert umgangssprachliche Formen und einige ruhrdeutsche Signale (ICH SA:ch da NIX zu, von unsern HAUSwirt, war damals nich BEI da, kein Schritt WEIter (=Nom. statt Akk.)).

Allerdings ändert sie mitten in der Erzählung ihre prosodischen Signale: Ihre Erzählung beginnt in Z. $375 \mathrm{ff}$ mit normaler kräftiger Stimme und relativ kurzen Akzenteinheiten, die der Erzählung einen 'pointierten', 'bestimmten' und 'energischen' Ton verleihen. Der Erzählhöhepunkt ist zusätzlich durch die Lautstärke hervorgehoben:

\begin{tabular}{|c|c|}
\hline $\begin{array}{l}\text { da war } \\
\text { poLteramd von seiner } \\
\text { TOCHter }\end{array}$ & $\begin{array}{l}(0.9) \\
(0.4)\end{array}$ \\
\hline $\begin{array}{l}\text { un da hat ihr } \\
\text { MANN wohl mit ner } \\
\text { ANdern ge } \\
\text { TANZT }\end{array}$ & $\begin{array}{l}(1.0) \\
(0.7) \\
(0.5) \\
(0.5)\end{array}$ \\
\hline $\begin{array}{l}\text { ICH weiß es nich } \\
\text { war damals nich } \\
\text { BEI da. }\end{array}$ & $\begin{array}{l}(0.7) \\
(0.7) \\
(0.8)\end{array}$ \\
\hline $\begin{array}{l}\text { (rel. lout) } \\
\text { GING se } \\
\text { LO: } \mathrm{S}^{*}\end{array}$ & $\begin{array}{l}(0.4) \\
(0.4)\end{array}$ \\
\hline
\end{tabular}

In der Folgeäußerung

$\begin{array}{ll}\text { un dann } & (0.5) \\ \text { HAT s sich wit: ihra } & (0.9) \\ \text { MANN wohl } & (0.6) \\ \text { AUCH gehabt } & (0.6) \\ \quad \text { un dann } \text { ä: h } & \end{array}$

gerät sie aber offenbar zweimal ins Stocken, vielleicht nachdem sie realisiert hat, daß B's Bestätigungen zeigen, daß er den Verlauf des Ereignisses kennt und deshalb vermutlich auf etwas anderes als Tratschen hinaus will. Ab Z. 383 jedenfalls ist K's Sprechweise abrupt schneller und leiser; der gesamte noch folgende Teil der Erzählung wird als eine Einheit präsentiert, die kontinuierlich leiser wird und bis zum Schluß auf einer kontinuierlich fallenden globalen Tonhöhe liegt. Die Akzenteinheiten sind hier länger mit durchschnittlich mehr als einer Sekunde Zeitdauer zwischen den Akzenten; man könnte sich die langen Takte hier gut aufgeteilt denken in jeweils 2-3 kürzere, um sich die Länge dieser Takte im Vergleich vorzustellen:

is die Poli

ZEI gerufn worn

- un da 


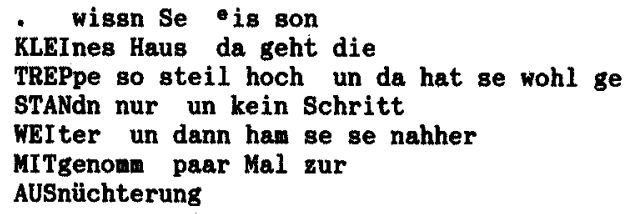

Diese Äußerungen mit längeren Akzenteinheiten wirken weniger 'pointiert' und 'bestimmt' und weniger 'energisch', eher 'kraftloser', 'auslaufend' und 'beiläufig'. Insgesamt hört sich K's Stimme wieder an wie vor ihren Erzählungen und so wie sie vorher geschnieft hatte schnieft sie auch am Ende dieser Erzählung in Z. 383 wieder $^{11}$.

Die Interpretation dieser veränderten prosodischen Signale in diesem Gesprächsausschnitt ist nicht eindeutig und es ergeben sich zwei Interpretationsmöglichkeiten: Entweder $\mathrm{K}$ läßt hier ihre Erzählung beiläufig auslaufen, weil sie vielleicht an der Gültigkeit des alltagsweltlichen Interaktionsrahmens zu zweifeln beginnt und mißtrauisch bei $B$ einen strategischen Zug erahnt - eine Interpretation, die ich plausibler finde und weiter verfolgen werde. Oder $\mathrm{K}$ setzt diese Signale als narratives Stilmittel zur Spannungssteigerung ein, ist damit aber wenig erfolgreich.

Neben der 'auslaufenden' Prosodie sprechen für die Interpretation, daB $\mathrm{K}$ ihre Erzählung eher beiläufig auslaufen lassen und herunterspielen will, v.a. noch ihre Wortwahl: Während sie den Höhepunkt in Z. 379 (rel.laut) GING se LO:S, mit dem sie an B's Wortwahl vorher anknüpft, noch eher 'hochspielt', ist die Formulierung sich mit ihrm $M A N N$ wohl $A U C H$ gehabt bereits wesentlich vager; dann wird die Frau GG in Z. 385 als nur noch drohend bei der Treppe stehend dargestellt und schließlich erscheint angesichts der AUSnüchterung die Episode gar als relativ harmloser "Ausrutscher" einer betrunkenen Frau. Vom Brotmesser ist zumindest nirgendwo mehr die Rede.

Im Rahmen dieser Interpretation wäre die Sequenz wissn $S e e_{i s}$ son KLEInes Haus da geht die TREPpe so steil hoch un da hat se wohl gestandn nur un kein Schritt WEIter (Z. 383-385) als Einschub zwecks Konkretisierung und Präzisierung dieser Episode mit Wiedergabe direkter Rede zu verstehen. Danach würde dann der Wiedereinstieg in die Darstellung der unterbrochenen Ereignisabfolge mit nahher signalisiert.

Eventuell spricht auch für diese Interpretation, daß B während des ganzen zweiten Teils der Erzählung keine Rezeptionssignale mehr liefert und auch sonst den zweiten Teil der Erzählung nicht erkennbar als Erzählung behandelt.

Während bis hierher also $B$ und $K$ beide in aufeinanderfolgenden Turns ihre Sprechstile fast sukzessive weiter in Richtung Ruhrdeutsch und informelle Unterhaltung verschoben haben, konstituiert B in Zeile 389-390 mit der Äußerung

die

$\begin{array}{ll}\text { SOLL auch } & (0.4) \\ \text { sehr eifersüchtich auf } & (0.9) \\ \text { SIE sein ne } & (0.7)\end{array}$

11 Dies zeigt, daß auch Vokalisierungen wie Schniefen hier und andere parasprachliche und non-verbale Gesten als Klammern und Kontextualisierungshinweise verwendet werden. 
einen abrupten Stilwechsel zurück zur Hochsprache und zu wiederum pointierterer Akzentuierung mit kurzen Akzenteinheiten und signalisiert damit einen Wechsel zurück zur formellen Interaktionsmodalität innerhalb des institutionellen Rahmens. Hier wird nun $\mathbf{K}$ auch wieder direkt mit sie adressiert.

In der Form der Thematisierung eines weiteren Gerüchts (die SOLL) knüpft B hier offenbar unvermittelt wieder beim Thema der früheren Verhörphase an, nämlich K's Aufenthaltsort und Lebenswandel, und besteht auch nach 0.9 Sekunden Pause auf diesem Thema. Der Sprechstil signalisiert, daß nun wieder die institutionellen Interpretationsschemata gelten und Informationen von und über $\mathrm{K}$ quasi institutionell gegen sie verwendet werden können, wenn auch nur im Hinblick auf ihre soziale Kategorisierung. Der von B initiierte Rahmenwechsel zum Tratsch erscheint retrospektiv als strategischer Zug. Er zeigt, daß - wie vielleicht $K$ ja auch schon in Rechnung gestellt hat - kurzfristige lokale Modalitäten- und Rahmenwechsel die übergeordnete Gültigkeit des globalen behördlichinstitutionellen Interaktionsrahmens, wie er durch das SETTING vordefiniert ist, zumindest für B nicht außer Kraft setzen.

Dieses Mal folgt $\mathrm{K}$ dem von B konstituierten Sprechstilwechsel jedoch nicht, sondern versucht, sowohl sprechstilistisch wie auch thematisch das von B initiierte Schema zu verlassen. Dies zeigt aber, daß sie den von B signalisierten erneuten Rahmenwechsel sehr wohl wahrgenommen hat, ihn aber nicht ratifiziert.

\section{Nun zum zweiten Gesprächsausschnitt:}

Während im ersten Gesprächsausschnitt Sprechstilwechsel vor allem auch zur Konstitution formeller und informeller Interaktionsmodalitäten und zur Kontextualisierung von Aktivitäten in lokale Interaktionskontexte strategisch verwendet und ausgenutzt werden, ist im zweiten Gesprächsausschnitt eine solche strategische Verwendungsweise nicht erkennbar. Dort werden im Rahmen kooperativer Interaktion unterschiedliche Interaktionsmodalitäten bzw. unterschiedlich modalisierte Aktivitäten in rascher Aufeinanderfolge unterschieden. Diese Verwendungsweise stützt die bisherige Analyse und zeigt darüber hinaus, daß Varietätenveränderung und Prosodie unabhängig voneinander funktionieren.

Bei der Analyse des zweiten Transkripts möchte ich mich nun auf den Vergleich einiger ausgewählter Turns und Äußerungen konzentrieren, in denen dort Stilveränderungen von $K$ und $B$ konstituiert werden.

$\mathrm{Zu}$ Beginn des Gesprächsausschnitts verwenden $\mathrm{B}$ und $\mathrm{K}$ eine unmarkiert umgangssprachliche Sprachvarietät und relativ kurze Akzenteinheiten, wenn sie den Sachverhalt etablieren, daB $\mathrm{K}$ von einem Bekannten einen Herd für ihre neue Wohnung bekommen kann, sich jedoch nicht nach dem Preis erkundigt hat. Folgende Akzenteinheiten kommen hier vor:

B sagt in Zeile 573:

DER hat für
SIE son: $\quad\left(\begin{array}{l}(0.4) \\ \text { HERD }\end{array}\right.$


Daran anschließend formuliert K - nach einer Selbstkorrektur mit Bezug auf den Rhythmus ihrer Äußerung (siehe Zeile 574) - eine Kurzdarstellung der Hintergründe dieses Herdes ebenfalls mit unmarkierter umgangssprachlicher Varietät und kurzen Akzenteinheiten:

$\begin{array}{ll}\text { DIE ham } & (0.3) \\ \text { HEI zung ge } & (0.6) \\ \text { KRICHT un ham } & (0.6) \\ \text { ALles } & (0.3) \\ \text { RAUSgeworfn } & (0.6) \\ \text {. dann } & (0.6) \\ \text { ne } & \end{array}$

Diese Äußerungen klingen insgesamt 'faktisch' und 'sicher'; der Sachverhalt wird als behördlich relevanter Sachverhalt besprochen. Und auch B's Entgegnung in Zeile 579-581 enthält vier kurze Akzenteinheiten, bevor er sie mit einem etwas längeren unakzentuierten Nachtrag nach der letzen Akzentsequenz auslaufen läßt:

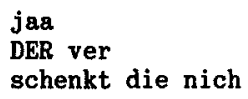

äh der

AA is ja

froh wenn die

hier sind hadder wir gesacht

Mit den kürzeren Akzenteinheiten kontrastiert dann jedoch K's Rhythmus in den Zeilen 585-587:

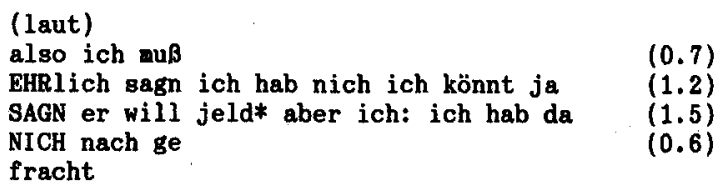

Neben den merklich längeren Akzenteinheiten verwendet $K$ hier auch einige Kernmerkmale einer markierten umgangssprachlichen Varietät, nämlich jeld und da ... nach. Insgesamt klingt die Äußerung aufgrund der Veränderung dieser beiden Parameter Sprechrhythmus und Varietät weniger 'pointiert' und 'beiläufiger' und informeller als die vorherigen Äußerungen. Die so kontextualisierte Äußerung ist ein Kommentar zur weiteren Erläuterung des Hintergrundes zum thematischen herd. In der metakommunikativen Einleitung zu dieser Äußerung expliziert $\mathbf{K}$ den beziehungsbezogenen, imagepflegenden und selbstdarstellenden Zweck dieses eher als 'persönliche Bemerkung' präsentierten Kommentars: Sie versucht sich hier als ehrliche Person darzustellen und unterstreicht ihre 
dargestellte Ehrlichkeit noch durch die Explikation möglicher Handlungsalternativen. Dies kann sie aber aufgrund der in unserer Kultur herrschenden Interkationsregeln, -normen und Präferenzstrukturen am ehesten mit einer als 'beiläufigen Hintergrundkommentar' präsentierten Äußerung erreichen (vgl. etwa eine interpretierende Umschreibung wie: 'es ist ja eigentlich selbstverständlich, aber ich wollte es sicherheitshalber noch einmal gesagt haben'); dieselbe Äußerung 'pointierter' und 'weniger beiläufig' mit kurzen Akzenteinheiten würde vielleicht eher als Verteidigung gegen einen unterstellten Angriff oder als (eine ihrer Rolle hier nicht angemessener, zu) deutlicher 'Heilungsversuch' eines unerwünschten Images und einer nicht-befriedigenden Interaktionsbeziehung (man erinnere sich auch noch einmal an die Interaktion im ersten Transkriptausschnitt!) interpretiert. Indem sie aber zugleich diese Äußerung mit dem 'Hintergrundkommentarston' mit erhöhter Lautstärke präsentiert, erreicht sie offenbar auch deren Unüberhörbarkeit.

B reagiert mit einer Ratifizierung des Hintergrundkommentars. Er folgt K's Sprechstilwechsel jedoch nur auf der Ebene der Sprachvarietät: Neben zahlreichen unmarkiert umgangssprachlichen Formen wählt er auch das markierte Signal dat in Zeile 589 und signalisiert damit offenbar ebenfalls eine Varietätenverschiebung in Richtung markierter Umgangssprache oder Ruhrdeutsch. Seine Akzenteinheiten hingegen sind kurz:

$\begin{array}{ll}\text { jojo da } & (0.4) \\ \text { BRAUchn se au nich } & (0.4) \\ \text { dat ding könn se } & (0.5) \\ \text { So mitnehm } & (0.3) \\ \text { HADder ge } & \\ \text { sacht } & \end{array}$

Diese Äußerungen, in denen B K's vorher durchgeführte und dargestellte Aktivitäten ratifiziert und kooperativ unterstützt, bewirken damit zum einen eine Annäherung an K's Sprechweise, scheinen mit den kurzen Akzenteinheiten jedoch zugleich auch diesen Aktivitäten einen nicht-beiläufigen und das (Image-)Problem der K ernst nehmenden Charakter zu verleihen.

Eine Sprechstilveränderung, mit der B auf die Interaktionsbeziehung zwischen B und $K$ bezogene informellere Sequenzen absetzt von formellen Handlungsanweisungen an $K$ findet sich auch in der Instruktionssequenz in den Zeilen 599-616. Diese besteht insgesamt (1) aus einem Wenn-Teil der Instruktion (Zeilen 599-603), (2) einer auf die Interaktionsbeziehung bezogenen Nebenbemerkung, die vor allem durch einen veränderten Rhythmus und eine auf gleichbleibend mittlerer Tonhöhe verbleibende Intonationskontur vom Wenn-Teil abgesetzt sind, thematisch jedoch unmittelbar an diesen anschließt (Zeilen 605-607), (3) einen Dann-Teil der Instruktion (Zeilen 609-612) und schließlich (4) aus einer Plausibilisierung, in der B. seine Handlungsvorstellungen abgrenzt gegen eine der $\mathbf{K}$ zugeschriebene mögliche Handlungsvorstellung:

(1) Wenn-Teil der Instruktion:

is auch kein prob

LEM wenn sie jetz also 


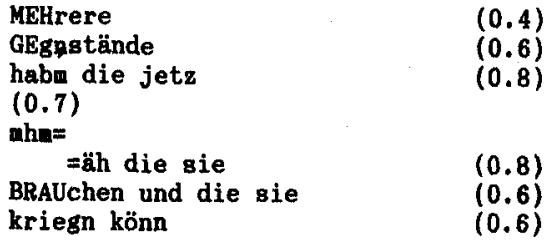

(2) Nebenbemerkung:

KOStplos oder gegn geringn (1.2)

PREIS dat aüssn wa dann no (0.9)

AUShandeln

(3) Dann-Teil der Instruktion:

$\begin{array}{ll}\text { RUF ich den } & (0.4) \\ \text { EILbotn an un und } & (0.5) \\ & (0.7) \\ \text { SAge wir gind be } & (0.6) \\ \text { REIT die und die } & (0.7) \\ \text { KOSty für den trans } & (0.8) \\ \text { PORT zu ubbernehy } & (0.8)\end{array}$

(4) Plausibilisierung:

$\begin{array}{ll}\quad \text { aber ich } & (0.4) \\ \text { KANN nich für } & (0.5) \\ \text { EIY } & (0.3) \\ \text { HERD den } & (0.4) \\ \text { EILboty } & (0.5) \\ \text { DAhin schicken } & (0.5) \\ & \\ \quad \text { un dann } & (0.2) \\ \text { kom se nächste mal mit n } & (0.8) \\ \text { STUHL un den } & (0.5) \\ \text { hol ich dann in } & (0.6) \\ \text { HALstade mit n eilbot } & (1.4)\end{array}$

In dieser Sequenz werden die Instruktionsteile (1) und (2) und der erste Teil der Plausibilisierung vorwiegend in einer relativ hochsprachlichen Varietät und mit relativ kurzen Akzenteinheiten realisiert. Diese Teile wirken 'bestimmt', 'belehrend', die Instruktion 'einhämmernd'. K reagiert mit Rezeptionssignalen.

Davon unterscheidet sich vor allem die Nebenbemerkung als Teil (2) dieser Sequenz. Im Laufe der Nebenbemerkung verschiebt B seine Sprachvarietät in Richtung markierter Umgangssprache (dat und einige unmarkierte Signale); die Akzenteinheiten sind merklich länger als in seinen Äußerungen vorher und nachher. Eher informell und nebenbei 'beiläufig' räumt $\mathbf{B}$ hier die Möglichkeit ein, mit $\mathbf{K}$ ihren Preisspielraum auszuhandeln, wenn sie seine Instruktionen ausführt, und stellt damit eine Kooperation in Aussicht. 
Die Teile (3) und (4) dieser Sequenz werden zugleich intonatorisch von den vorhergehenden Teilen abgesetzt: Statt der zuvor verwendeten fallenden ' $Y$ ' Akzente finden sich nun Akzentsequenzen, in denen fallende ' $Y$ ' und steigende '/' Akzenttypen kombiniert werden. Einerseits werden dadurch diese Teile als Einheit von den vorherigen abgesetzt, andererseits erscheinen diese beiden Teile als selbst untereinander kohäsiv verbundene Teile. Gegenüber den eher einförmigeren $F(\backslash \backslash \backslash$ o.ä. Akzentsequenzen aus nur fallenden Akzenttypen, die z.B. von Essen 1956 als angemessene Intonationskonturen u.a. für öffentliche Vorträge empfiehlt, wirken die in den Teilen (3) und (4) verwendeten variationsreicheren Akzentsequenzen wie $S(\backslash /$ ) oder $S(\backslash / / \backslash)$ usw. (siehe Transkript 2) bereits weniger 'belehrend' und formell.

Am Ende der Sequenz im letzten Teil der Nachbemerkung verwendet B einige markiert ruhrdeutsche Signale (Akkusativ statt Dativ in mit $n+$ Nomen mask sg) und ganz zum Schluß eine lange Akzenteinheit von ca 1.4 Sek. Dauer. Bei dieser letzten Äußerung handelt es sich dann bereits um eine etwas übertriebene und damit auch in ihrer $\mathrm{Zu}$ schreibung an $\mathrm{K}$ bereits unplausible Explikation einer Handlungsweise, von der $\mathrm{B}$ sich absetzt. Hier wird also der propositionale Gehalt dieser Äußerung quasi durch die Sprechstilveränderung neutralisiert: B treibt lediglich eine unhaltbare Handlungsvorstellung durch Überziehung auf die Spitze, signalisiert aber bereits durch seinen informellen und 'beiläufigen' Sprechstil, daß er diese nicht ernsthaft der $\mathbf{K}$ unterstellt. K selbst, die sich im Laufe des Gesprächs gegen ungerechte Unterstellungen immer wieder zur Wehr gesetzt hat, scheint dies hier auch im explizierten Sinne zu verstehen und signalisiert explizit, daß sie nun verstanden hat, was sie tun soll, um ihre neue Wohnung bezugsfähig zu machen.

Auch in dieser Sequenz werden damit die Wahl und Verschiebung sprachlicher Varietäten und Prosodie verwendet, um formell und informell modalisierte Aktivitätsteile zu konstituieren und dem Interaktionspartner als solche erkennbar zu signalisieren. Beide Parameter funktionieren jedoch unabhängig voneinander; sie können zusammen verändert werden, jedoch auch durch Veränderung nur eines Parameters an unterschiedliche Vorgängerstrukturen kohäsiv anknüpfen.

\section{ZUSAMMENFASSUNG UND FAZIT}

Es dürfte deutlich geworden sein, daß und wie Interaktionspartner neben sprachlichen Formulierungen auf der 'Textebene' insbesondere sprachliche Varietäten und deren Verschiebung und Wechsel wie auch prosodische Signalisierungsmittel wie Rhythmus, Intonation, Lautstärke und Sprechtempo als sozial und interaktiv bedeutsame Mittel verwenden, um Interaktionsmodalitäten und -rahmen zu signalisieren und auszuhandeln. Im vorliegenden Gesprächsausschnitt wurden von den Gesprächspartnern v. a. Varietätenverschiebungen und unterschiedliche Akzentrhythmen als sprechstilkonstituierende Mittel verwendet, um mit ihren Äußerungen unterschiedliche lokal relevante Interaktionskontexte herzustellen und die damit verbundenen Interpretationsrahmen nahezulegen. Die damit hergestellten Sprechstile selbst werden als sehr flexible, aber sozial typisierte und interpretierte, interaktive Einheiten aufgefaßt; ihre konkrete Gestalt ist immer auch das Produkt interaktiver Aushandlungs- und Bedeutungszuschreibungs- 
prozesse im 'Mikrokosmos' des Gesprächs. Wenn die Signalisierung und Veränderung sprachlicher Varietäten eher im Hinblick auf die Konstitution und Veränderung von Gesprächsebenen und -modalitäten, hier im Bereich der Dimensionen Formalität-Informalität und institutionelle versus alltagsweltliche Interaktions- und Interpretationsrahmen, interpretierbar scheint, dann scheinen v.a. unterschiedliche Akzentrhythmen hier eher im Hinblick auf Dimensionen wie signalisierte konversationelle 'Bestimmtheit' und 'Pointiertheit' oder auch signalisierte 'Stärke' o.ä. interpretierbar zu sein: Kürzere Akzenteinheiten wirken offenbar 'pointierter' und die Äußerung enger fokussiert und 'bestimmt', 'energisch' oder auch 'belehrend' und in den "kommunikativen Vordergrund" gestellt; längere Akzenteinheiten wirken weniger 'pointiert', weniger 'bestimmt', oft 'auslaufend' und die Äußerung eher 'beiläufig', unter Umständen auch '(sich) zurücknehmend' und eher in den "kommunikativen Hintergrund" gerückt. Mit einem ikonischen Bild könnte grob analogisiert werden: Je kürzere Akzenteinheiten in Äußerungen, um so deutlicher versucht offenbar die Sprecherin oder der Sprecher, dem Rezipienten 'offensiv' und "vordergründig" ihre/seine Signale und Mitteilungen "ins Bewußtsein zu hämmern". Die Wahl und Veränderung sprachlicher Varietäten und prosodische Veränderungen können zusammen, jedoch auch unabhängig voneinander als Signale verwendet werden, um bestimmte Kontextualisierungen und lokal relevante Interpretationsrahmen nahezulegen und ggf. kohäsive Beziehungen im Gespräch herzustellen.'

Im vorliegenden Gespräch werden jedoch Sprëchstilveränderungen vorwiegend vom Institutionsvertreter initiiert. Das entspricht der allgemeinen Erwartung, daß in solchen institutionellen Kontexten der Institutionsvertreter die Definitionsmacht und das Recht zur Nutzung seines Handlungsspielraums hat, auch strategisch.

Andererseits zeigt sich hiermit natürlich auch, daß die Interaktionspartner offenbar ein Alltagswissen über die soziale Ordnung in solchen Gesprächen zugnunde legen, die hier offenbar reproduziert und kaum in Frage gestellt wird: Jedenfalls behauptet B seine Rolle als definitionsmächtiger Institutionsvertreter und $\mathrm{K}$ agiert in der Rolle der Abhängigen, die auch strategisch ausgetrickst werden kann. Hier gehen neben den lokalen Konstitutions- und Aushandlungsprozessen und -bedingungen in der Situation auch allgemeinere Interaktionsbedingungen mittels des Alltagswissens der Interaktionspartner über Rechte und Pflichten in der (institutionellen) Interaktion, Prestige und Image, Status und Rolle, Macht und Herrschaft, soziale und interaktive Normen usw. ein (vgl. hierzu insbesondere auch Gloy 1981). Deshalb bietet die Rekonstruktion der interaktiven und sozialen Bedeutung von Sprechstilen auch Anknüpfungsmöglichkeiten für eine Untersuchung der 'Reproduktion sozialer Verhältnisse im Mikrokosmos des, Gesprächs' bzw. der Manifestation gesellschaftlicher Makrostrukturen in konkreten Interaktionen im Rahmen weiterreichenderer soziolinguistischer oder soziologischer Forschungsansätze. 


\section{ANHANG}

Bei der Transkription des Gesprächsausschnitts wurden folgende Transkriptionskonventionen zugrunde gelegt:

Charakterisierung der Einheiten und Sprechweisen in der Textzeile:

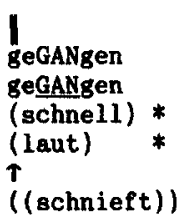

(0.8)

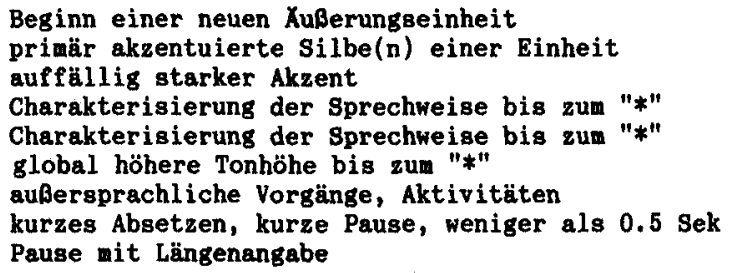

Intonationsnotation in der Intonationszeile:

Globale Einheiten:

$\mathrm{F}, \mathrm{S}, \mathrm{M}, \mathrm{H}, \mathrm{T}($ ) Globaler Tonhöhenverlauf der durch die

Klammern eingegrenzten kohäsiven Akzentsequenz

in der Klanner: $F=f a l l e n d, ~ S=s t e i g e n d$,

$M=m i t t e l, H=h o c h, T=t i e f ;$

H/F Kombination von globalen Angaben

(...) Folge von schwachen Akzenten

$[()()]$ zusammengesetzte Kontur

Akzentnotationen innerhalb von Klanmern:

Gipfel- und/oder fallender Akzent (früher "+")
Tal- und/oder steigender Akzent (früher "-")
Plateau- bzw. gleichbleibender Akzent (fr "=")
lokaler Tonhöhengprung nach oben bei einem
Akzent

Nachläufe nach der hinteren Klaner:

$1,1,-\quad$ unakzentuierte Nachläufe nach dem letzten

Akzent der Akzentsequenz mit noch veränderten

Tonhöhenverlauf: fallend, steigend,

gleichbleibend 


\section{LITERATUR}

Abercrombie, David (1964). Syllable quantity and enclitics in English. In: ders. et al. (eds.). In honour of Daniel Jones. London: Longman, 216-222.

Auer, Peter (1986). Kontextualisierung. Studium Linguistik 19, 171-188.

Bergmann, Jörg (1987). Klatsch. Zur Sozialform der diskreten Indiskretion. Berlin New York: de Gruyter.

Bolinger, Dwight (1986). Intonation and its parts. Stanford: Stanford University Press.

Couper-Kuhlen, Elizabeth/Auer, Peter (1988). On the contextualizing function of speech rhythm in conversation: question-answer sequences. Arbeitspapier Nr. 1 aus dem Projekt "Kontextualisierung durch Rhythmus und Intonation", Universität Konstanz, Januar 1988.

Dittmar, Norbert/Schlobinski, Peter (1988). Convergence, discourse and variation. In: P. Auer/A. di Luzio (eds.). Variation and convergence. Berlin etc.: de Gruyter, 157-175.

Erickson, Frederick/Shultz, Jeffrey (1982). The counselor as gatekeeper. New York etc.: Academic Press.

Essen, Otto von (1956). Grundzüge der hochdeutschen Satzintonation. Ratingen: Henn.

Gloy, Klaus (1981). Deutungsschemata des Sozialamts - Zur linguistischen Analyse von Institutionen als Konfliktherden. In: J. Klein/G. Presch (Hrsg.). Institutionen - Konflikte Sprache. Arbeiten zur linguistischen Pragmatik. Tübingen: Niemeyer, 87-125.

Gumperz, John J. (1982). Discourse Strategies. London: Cambridge University Press.

Halliday, Michael A. K. (1967). Intonation and grammar in British English. The Hague: Mouton.

Hymes, Dell H. (1979). Uber Sprechweisen. In: ders., Soziolinguistik. Eingel. und hrsgg. von Florian Coulmas. Frankfurt/M.: Suhrkamp, 166-192.

Kallmeyer, Werner/Keim, Inken (1986). Formulierungsweise, Kontextualisierung und soziale Identität. Zeitschrift für Literaturwissenschaft und Linguistik (LiLi) 64, 98-126.

Mihm, Arend (1985). Sprache an Rhein und Ruhr: dialektologische und soziolinguistische Studien zur sprachlichen Situation im Rhein-Ruhr-Gebiet und ihrer Geschichte. Stuttgart: Steiner-Verlag-Wiesbaden-GmbH.

Müller, Klaus (1984). Rahmenanalyse des Dialogs. Tübingen: Narr.

Pheby, John (1980). Intonation und Grammatik im Deutschen. Berlin: Akademie-Verlag, $1980^{2}$.

Quasthoff, Uta (1980). Erzählen in Gesprächen. Tübingen: Narr.

Reiffenstein, Ingo (1976). Primäre und sekundäre Unterschiede zwischen Hochsprache und Mundarten. In: Opuscula slavica et linguistica. Festschrift für Alexander Issatschenko, hgg. v. Heinz Dieter Pohl. Klagenfurt: Heyn, 337-347.

Reiffenstein, Ingo (1980). Zur Theorie des Dialektabbaus. In: J. Göschel/P.Ivic/K. Kehr (Hrsg.). Dialekt und Dialektologie. Wiesbaden: Steiner, 97-105.

Sandig, Barbara (1986). Stilistik der deutschen Sprache. Berlin: de Gruyter.

Schirmunski, Viktor (1930). Sprachgeschichte und Siedlungsmundarten. Germanisch-romanische Monatsschrift 18, 113-122 und 171-188.

Selting, Margret (1985). Ebenenwechsel und Kooperationsprobleme in einem Sozialamtsgespräch. Zeitschrift für Sprachwissenschaft 4/1, 68-89.

Selting, Margret (1987). Descriptive categories for the auditive analysis of intonation in conversation. Journal of Pragmatics 11, 777-791.

Selting, Margret (1989). Speech styles in conversation as an interactive achievement. In: Leo Hickey (ed.) (in press). The Pragmatics of Style. London: Routledge.

Selting, Margret (1989a). Intonation as a contextualization device: case studies on the role of prosody, especially intonation, in contextualizing story telling in conversation. Ms., erscheint in: P. Auer/A. di Luzio (eds.) (in prep.). Contextualization of Language. Amsterdam: Benjamins.

Streeck, Jürgen (1985). Die leichte Muse des gewöhnlichen Gesprächs, Über die Unterhaltungskunst einiger älterer Frauen. Manuskript. 